still are so keen. One of the best features of Race into Space is the attempt to explain the impetus in historical terms. The inspiration came from Konstantin Tsiolkovsky (1857-1935) who, in books such as $A$ Dream of Earth and $S k y$ (1895), foresaw nearly all of the events that have actually occurred. He advocated liquid-fuel engines, the staging of rockets, solar power and even stellar ships with closed ecocycles. Surprising as it may seem to those living in Western countries, where rocket enthusiasts were scorned until after 1940 , Tsiolkovsky was encouraged by the state; he was given a pension in 1918 , and

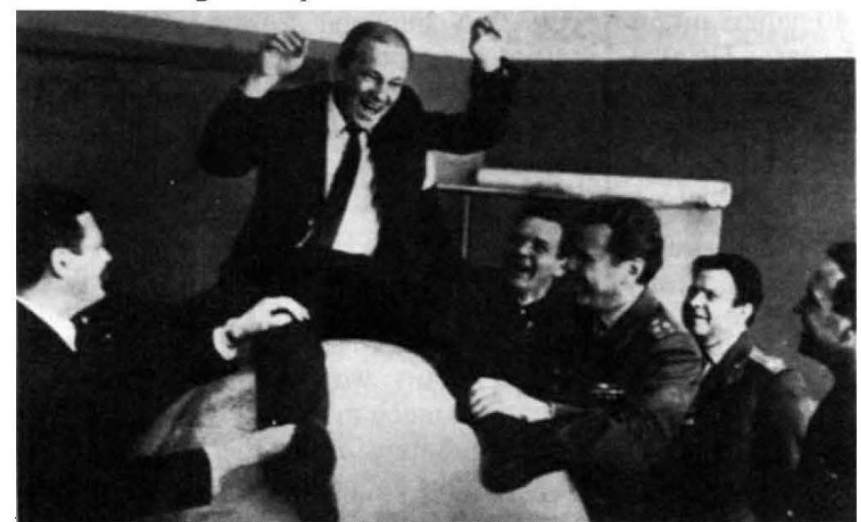

On top of the world - the cosmonauts involved in the Salyut 1 mission. The picture is reproduced from The Soviet Manned Space Programme by Phillip Clark, Publisher is Salamander, price is $£ 14.95$.

a meeting of the Academy of Sciences was held in his honour in 1932. His home was to become a place of pilgrimage. Scientific achievement (unsullied by the profit motive) was held dear by the Soviet government in the 1920 s and early 1930 s, when the engineer Frederick Tsander (1887-1933) constructed liquid-fuel rockets, the author Yuri Kondratyuk foresaw the future course of space exploration, and research institutes for jet propulsion and aerodynamics were established. "By the mid 1920s, all the major components of the Soviet space programme were in place", Harvey remarks, and it seems a defensible claim. But the programme ran into the sands, because of policy changes and Stalin's purges - among those imprisoned was Sergei Korolov (1906-1966), later the 'chief designer' of space launchers. After the Second World War, when the German V2 showed everyone what could have been done, Soviet rocket development went ahead strongly. The desire for military rockets was the driving force, but their possible use for space launching was quickly recognized, and exploited by Khruschev from 1954 onwards.

After this historical chapter, Race into Space becomes a detailed narrative, seen always in terms of rivalry with the United States, of Soviet manned spaceflight and lunar and planetary exploration. Only a few pages are devoted to the many other types of satellites that make up the great majority of the Soviet space launches.
There is very little about the photographic reconnaissance satellites, of which nearly a thousand have been launched, or about the many satellites for ocean surveillance, electronic ferreting and early warning. The systems of satellites for communications, meteorology and navigation are only glanced at, and there is scarcely a mention of the numerous spacecraft carrying scientific experiments.

The strength of the book is in its detailed accounts of the out-and-back journeys of the cosmonauts, all of whom are mentioned by name. Not all have been Soviet citizens: they have come from many different countries, ranging alphabetically from Bulgaria and Cuba to Syria and Vietnam (with Afghanistan as a recent addition). With so many flights to be covered, the details of the crews may seem almost as boring as a roster of bus drivers leaving and returning to their depot. Harvey recognizes this problem and tries to enliven the text with character-sketches of some of the cosmonauts and overdone dramatizations. For example, there is the story of the US astronauts arriving by airliner at "Star City': "out jumped the three Americans", we are told, and after this improbable gymnastic feat they drive in a minibus past "endless avenues" in this city surrounded by desert, "endless, flat and arid", with a river running through it. Wet or dry, the style is not flat, and the adjectives are as endless as the avenues or the desert. At the other extreme, the crew of Voskhod2, landing at night, had to endure "frosty breath from lurking shadowy forms" (these are wolves, not the welcoming party).

There are plenty of photographs, not of wolves, but chiefly of cosmonauts, who all look remarkably similar in space suits, especially as the quality of the photographs is poor. Also there are no diagrams in the main text, and the author is left with the unenviable task of trying to describe in words the complex design of Mir. All in all, the book does provide a useful, detailed account of Soviet manned spaceflight and lunar and planetary exploration, though the style may deter some wouldbe readers and minor inaccuracies are numerous.

Desmond King-Hele, 3 Tor Road, Farnham, Surrey $G U 97 B X, U K$, was formerly a Deputy Chief Scientific Officer at the Royal Aircraft Establishment, Farnborough. Among other books he is author of Satellites and Scientific Research (1960) and Satellite Orbits in an Atmosphere (1987).

\section{Arp's astronomical exotica}

\section{Michael Rowan-Robinson}

Quasars, Redshifts and Controversies. By Halton Arp. Interstellar Media, 2153 Russell Street, Berkeley, California 94705/ Cambridge University Press:1988. Pp.198. $\$ 22.20, £ 12.50$.

IN 1966 Halton Arp compiled one of the most fascinating catalogues of modern astronomy, the Arp Atlas of Peculiar and Interacting Galaxies. For nearly 20 years the remarkable objects in this catalogue were for the most part ignored by astronomers, who continued to think of galaxies as basically regular-shaped spirals or ellipticals. Now, in the past five years, interactions and mergers between pairs of galaxies have leapt to the forefront of the astronomical consciousness, partly because of the growth in sophistication of computer simulations of the dynamical interactions between galaxies, and partly because a higher than expected proportion of luminous infrared galaxies appear to be due to interactions or mergers.

But Arp will not be able to enjoy this tribute to his perspicacity. For as he was preparing his Atlas for publication, he noticed that some of the sources in the Third Cambridge Survey of Radio Sources appeared to lie in pairs on opposite sides of some of his peculiar galaxies. Because the recession velocities of the radiogalaxies and quasars with which these sources were identified were in general enormously larger than those of the "associated' peculiar galaxies, not only must the Hubble velocity-distance law break down in some circumstances but galaxies must be capable of spewing out whole galaxies and quasars.

This discovery took Arp down an extraordinary trail of quasars associated with nearby galaxies, companion galaxies with excess redshift, chains of galaxies with discordant redshifts, to quasars as members of the Local Group of galaxies. Because quasars were at that time so novel and incomprehensible many astronomers, myself included, were at first willing to keep an open mind about Arp's associations and to follow him at least a little way down the trail. Could the quasars, for example, like the nebulae before them, comprise more than one completely distinct kind of object?

Gradually, the possibility of there being any serious basis to Arp's claims dissolved. Quasars were found to be situated in galaxies and were often found to be in groups or clusters of galaxies which had the same redshift as the quasar. Statistically controlled surveys for quasars showed them to be distributed homogeneously 
over the sky. The Hubble law has been confirmed with ever-improving precision.

Today, Arp is left bitter and combative, with only a handful of astronomers such as Fred Hoyle and Geoff Burbidge taking him seriously. In this book he collects together all the best examples of his wondrous phenomena and an unimpressive and unconvincing assembly it makes. The 'lines' and 'concentrations' of quasars appear no more unusual than would be expected by chance, given that Arp has worked extremely hard to find these examples. The high-redshift galaxies near foreground objects always look just like more distant, background objects. Every so often Arp stumbles on some real phenomenon, such as the interactions of his 1966 Atlas or the optical jet of M87, but even then his explanation of it always has to be totally bizarre.

At one point Arp resorts to a rather sad piece of sophistry. Discussing the galaxies of Stephan's Quintet he remarks that a supernova had been discovered in 1971 in NGC 7319, the galaxy in the group which has an anomalously high redshift. Rather than accept that the faintness of the supernova supported a higher distance for the high-redshift galaxy, Arp concludes that: "stars in the anomalously redshifted systems simply do not get as bright as in galaxies of lower intrinsic redshift". This is sad because in 1961 Arp was the first to apply the Baade-Wesselink expansion parallax method to supernovae, and so could be called the father of the supernova distance method.

Arp claims that he has been harshly treated and kept off telescopes and out of astronomical journals. Yet he has managed to publish over 40 papers on his exotica over the past 20 years. He was president of the Astronomical Society of the Pacific from 1980 to 1983 , and has received a sprinkling of prizes and awards. This book illustrates the maxim that an obsession can ruin your whole life.

Michael Rowan-Robinson is Professor of Astrophysics in the Astronomy Unit, Oueen Mary College, Mile End Road, London E1 $4 N S, U K$

\section{The universal rover}

\section{Owen Gingerich}

The World Within the World. By John D. Barrow. Clarendon: 1988. Pp. 398. £20, $\$ 24.95$.

How does the uniformity of nature depend on the quantization of energy levels? I can only admire a 'popularization' that has the ingenuity to ask such a question. Here John Barrow, an astronomer at the University of Sussex, not only asks the question, but provides a notably clear answer: if the electrons orbiting protons could have any arbitrary speeds, then each hydrogen atom would be different, and "the uniformity of Nature an unrealized idealization".

In this wonderfully wide-ranging excursion, Barrow has brought a freshness of descriptive metaphor to some of the more arcane topics of modern physical science such as the Einstein-Podolsky-Rosen paradox, gauge theories, chaos, superstrings and the inflationary universe. Barrow explores not only a wide gamut of topics in the zone between physics and astronomy, but he wrestles with their philosophical contexts as well. His "world within the world" is the submicroscopic quantum framework within the largescale cosmos, so strangely incompatible with the relativistic picture of the vast astronomical Universe and yet so essential to its understanding. As he describes the march of science, he circles around a far more difficult question: are there really laws of nature out there to be discovered, or are we just imposing our own human
History of Time. Hawking applies a coordinate transformation to the singularity of the Big Bang, expands the time coordinate back to infinity, and glibly announces an originless Universe, asking "What place, then, for a creator?". Barrow, in contrast, discusses the question of singularities at some length, and concludes that until we better understand the nature of time, we will really not know how to answer those who ask whether the Universe is of finite or infinite age.

Although it achieves a sophisticated level of popularization, Barrow's World is nevertheless an uneven book. The conversation between a mathematical intuitionalist and a platonist is a confusing detour, and Barrow's road map of philosophical routes is too terse to set his readers onto clear paths. In his assessment of Thomas Kuhn's paradigmatic structure of scientific explanations, and elsewhere, he subtly suggests that the highest philosophical calling of a physicist is to be a realist. Yet his sympathetic discussion of the 'many worlds' interpretation of quantum mechanics, his description of multiple dimensions of the Universe, and his emphasis on metaphysical and aesthetic criteria as guides to theoretical speculation seem to make him far more the idealist.

Indeed, after Barrow's rewarding journey to the limits of space and time, his conclusion is not all that unexpected:

[these are] extraordinary problems, and they possess extraordinary solutions which will require extraordinary methods to coax from the Universe. If our methods ultimately fail, then any boundary between fundamental science and metaphysical theology will become increasingly difficult to draw. Sight must give way to faith. Confronted with an emotionally satisfying mathematical scheme which is 'simple' enough to command universal assent, but esoteric enough to admit no means of experimental test then, closeted within our world within the world, we might simply have to believe it.

Owen Gingerich is a Professor in the HarvardSmithsonian Center for Astrophysics, 60 Garden Street, Cambridge, Massachusetts 02138 , USA the sleight of hand that characterizes Stephen Hawking's best-selling A Brief

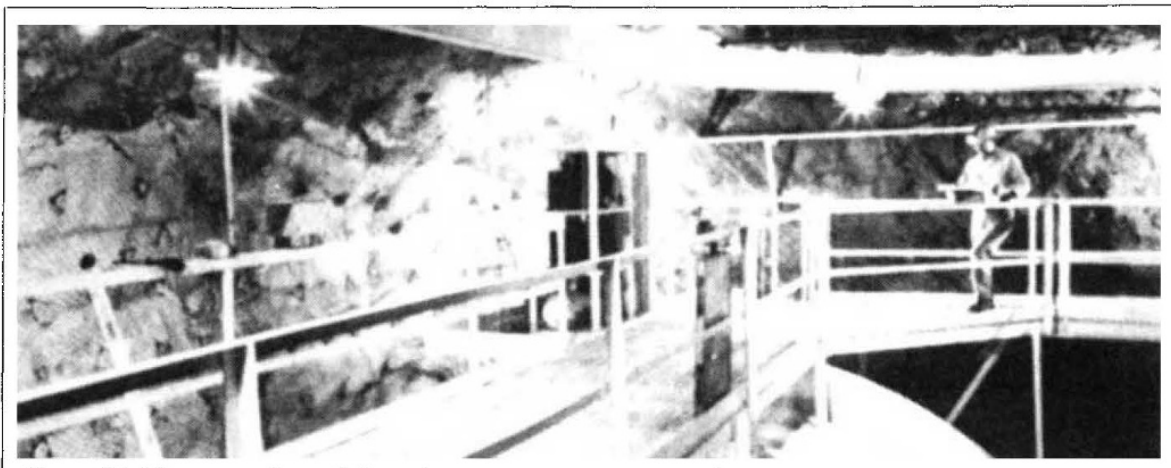

Deep thinking - a view of the solar neutrino experiment located at a depth of 4,850 feet in the Homestake gold mine. The picture is reproduced from Particle Physics: A Los Alamos Primer edited by N.C. Cooper and G.B. West, published earlier this year by Cambridge University Press. Price is hbk £30,\$49.50; pbk £9.95, \$14.95. 